\title{
Maximum entropy estimation of the bubble size distribution in fluidized beds
}

\section{Sobrino ${ }^{\mathrm{a}, *}$, J.A. Almendros-Ibáñez ${ }^{\mathrm{b}, \mathrm{c}}$, D. Santana ${ }^{\mathrm{a}}$, C. Vázquez ${ }^{\mathrm{d}}$, M. de Vega ${ }^{\mathrm{a}}$}

a Energy Systems Engineering (ISE), Department of Thermal Engineering and Fluid Mechanics, Universidad Carlos III de Madrid, Spain

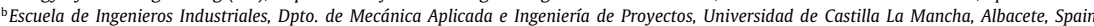
EScuela de Ingenieros Industriales, Dpto. de Mecánica
${ }^{\circ}$ Renewable Energy Research Institute, Albacete, Spain

${ }^{\mathrm{D} D i s p l a y}$ and Photonics Applications, Department of Electronic Technology, Universidad Carlos III de Madrid, Spain

\begin{abstract}
A B S T R A C T
This work presents a new methodology, based on the maximum entropy method, to obtain bubble characteristics in fluidized beds. The probability distributions (PDF) of bubble pierced length and velocity are obtained applying the maximum entropy principle to experimental measurements. In addition, the bubble diameter distribution has been inferred from experimental pierced length measurements. This method is applied to characterize bubbles in fluidized beds for the first time and the most general bubble geometry, a truncated spheroid, is considered. The distance between probes, $s$, which is the minimum pierced length that is possible to measure accurately using intrusive probes, has been introduced as a constraint in the derivation of the size distribution equation.

The maximum entropy method is applied to experimental measurements of bubble characteristics carried out using optical and pressure probes in a three-dimensional fluidized bed of Geldart B particles. Results on bubble size obtained from pressure and optical probes are very similar, although optical probes provide more local information and can be used at any position in the bed. The maximum entropy principle has been found to be a simple method that offers many advantages over other methods applied before for size distribution modeling in fluidized beds.
\end{abstract}

\section{Introduction}

Bubble characteristics, as bubble size, bubble shape, bubble ascending velocity and emulsion expansion are important parameters in the design and analysis of fluidized beds since they affect their performance as reactors. As bubbles are not directly observable, there have been a number of techniques developed to study the bubble properties in fluidized beds, some of them reviewed by Cheremisinoff (1986), Yates and Simons (1994) or Werther (1999).

Non-intrusive techniques such as X-ray (Kai et al., 2000) or magnetic resonance imaging (Müller et al., 2006) provide valuable information on bubbles in three-dimensional fluidized beds. However, they are expensive and limited to small equipment. Moreover, they exhibit a limited spatial or temporal resolution. Although disturbing the process to some degree, submerged probes are applicable to equipment and processes of virtually any size. As an example, capacitance probes have been used in fluidized beds with some success (Werther and Molerus, 1973), although they must be calibrated for every fluid-solid system and operating conditions. Optical probes

* Corresponding author. Tel.: +34916248464; fax: +34916249430

E-mail address: csobrino@ing.uc3m.es (C. Sobrino). have been widely used to measure particle velocity and particle concentration in three-dimensional fluidized beds. Some of them have been also adapted to detect the passage of bubbles (Ishida and Shirai, 1980; Hatano and Ishida, 1981). The procedure to obtain the bubble parameters is usually the same (Yasui and Johanson, 1958): two transmission probes are positioned one above the other spaced apart a variable short distance. The bubble rise velocity is estimated from the time lag between the signals from the two probes and their separation. The characteristic length associated with a bubble passage is calculated as the product of the bubble rise velocity and the time length of the light pulse transmitted by each bubble.

The interpretation of differential pressure measurements to determine the bubble parameters has received considerable attention because of its potential application in industrial fluidized beds. Sitnai (1982) first suggested the use of differential pressure measurements for the determination of bubble characteristics. He compared experimental measurements with simulated pressure gradient signals based on the theoretical pressure field around a bubble in a fluidized bed according to Davidson's model (Davidson and Harrison, 1963). Dual probes have also been used to detect bubble passage in a gas sampling system (Littman and Homolka, 1973) and in freely bubbling beds (Chan et al., 1987; Dent et al., 1989; Venkata Ramayya et al., 1996). 
The pierced length determination from dual submersible probe measurements in multiphase systems is the main time-consuming part of the diameter distributions inference processes. Then, the developing of new methods that minimize the required samples for the determination of the diameter distribution is an important goal in solid-gas-liquid systems. The inference of the bubble size distribution from the pierced length distribution obtained from experimental measurements is a well known problem addressed several years ago (Werther, 1974b). The chord length distribution $(P(y))$ is related with the bubble size distribution $(P(D))$ through the conditional probability of the pierced length given a bubble diameter $(P(y \mid D))$ as follows:

$P(y)=\int_{0}^{D_{\max }} P(y \mid D) P(D) d D$

where $P(D)$ is an unknown function to be solved whereas $P(y)$ can be obtained from measurements and $P(y \mid D)$ is inferred from geometrical relationships between $y$ and $D$ (Werther, 1974b). Eq. (1) is known as Fredholm integral equation of the first kind and it is common knowledge that it is an ill-conditioned problem. To overcome this problem several methods have been proposed, most of them by using a backward transformation. The first attempts to obtain the bubble size distribution by using a numerical backward transformation (Clark and Turton, 1988; Turton and Clark, 1989) showed a high dependence of the results on the discretization and on the sample size. On the other hand, Herringe and Davis (1976) deduced the nonlinear differential equation that describes the aforementioned problem. This differential equation is used to obtain the analytical backward transformation. Liu and Clark (1995) and Clark et al. (1996) obtained a parametric analytic solution of the problem assuming that the bubble size distribution follows a Gamma or a Rayleigh distribution. Then, for this method the distribution must be aprioristically fixed. Later, using the Parzen window, Liu et al. (1996, 1998) and Santana and Macías-Machín (2000) obtained a non-parametric analytical backward transformation. This method needs an iterative process to obtain the optimum window width. Although the method does not need to assume a distribution shape a priori, it requires a larger sample size to obtain a reliable bubble size distribution. It has been shown that the backward transformation is an unstable problem for all the aforementioned methods. Recently, Santana et al. (2006) reported a different method that avoids the use of the backward transformation. They found a semiparametric solution of the problem by applying the maximum entropy method and applied it successfully to gas-liquid systems assuming an elliptical bubble shape. In the maximum entropy method only several moment estimations are needed to determine the bubble size distribution and the raw moments estimation is a more affordable problem than the backward transformation. Moreover the sample size needed to obtain a reliable distribution is smaller than in the other methods. Santana et al. (2006) successfully applied this method to gas-liquid systems assuming an elliptical bubble shape. In their experiments air bubbles were injected in a water tank and image analysis was used to measure bubble diameter, velocity and angle of attack while the pierced lengths were synthetically generated. Other researchers, following a different approach, have used the statistics of pressure fluctuations to deduce the bubble size distribution in fluidized beds. For example, Bai et al. (2005) obtained the approximate shape of the void size distribution from pressure fluctuations measurements in beds working with group A and group $B$ particles.

In the present work, the maximum entropy method is used to characterize bubble size and velocity in fluidized beds for the first time. The bubble geometry considered is a truncated spheroid and the method is modified in order to introduce the effect of the sampling probe. Pierced lengths smaller than the probe size cannot be measured due to the finite size of the probe. This fact has an important effect that must be taken into account in the estimation of the bubble size distributions.

The main advantages of the proposed method are that the distribution shape does not have to be pre-established, the number of samples required is lower than in other methods and the backward transformation procedure is avoided. Moreover, although the distribution should match a certain number of moments obtained from the experimental measurements, the number of moments required does not need to be known a priori.

The first objective of this work is to build a method based on the maximum entropy distribution estimation for the conversion of pierced length into bubble diameter in bubbling fluidized beds. The second objective is to compare the performance of a plastic dual optical fiber probe (OFP) operating in the reflection mode (Vázquez et al., 2007) and a dual differential pressure probe for the determination of bubble velocity and pierced length in bubbling fluidized beds. The range of applicability of each technique for inferring bubble size distribution from the probe signals is analyzed. The signal processing needed to obtain bubble properties from the pierced time duration and the time lag between probes is explained.

\section{Modeling the bubble size distribution using the maximum entropy method}

In this paper the maximum entropy method (Jaynes, 1957) is used to obtain the probability density function (PDF) of the bubble velocity, bubble pierced length and bubble size in bubbling fluidized beds, from experimental measurements of the bubble pierced length and bubble velocity.

\subsection{Maximum entropy method}

Santana et al. (2006) applied the entropy principle of Shannon to obtain the bubble size, surface and volume distributions in air-water flows from the moments inferred from pierced length measurements. Intuitively, this method consists in, given a collection of constraints that an objective function has to satisfy, choosing a function which is consistent with all the constraints, but otherwise, being as uniform as possible. In other words, model all that is known and assume nothing about what is unknown. Following the formulation of Sellens and Brzustowski (1985), the probability distribution is the function that maximizes the Shannon entropy

$$
\begin{aligned}
& \max _{P(x)} \int_{x \in \Delta}-P(x) \ln (P(x)) d x \\
& \text { constrained to } \int_{x \in \Delta} f_{i}(x) P(x) d x=\left\langle f_{i}\right\rangle, \quad i=1,2, \ldots, n
\end{aligned}
$$

and also constrained to the normalization condition

$$
\int_{x \in \Delta} P(x) d x=1
$$

When the entropy is maximum, subjected to the constraints, the resulting $P(x)$ is the least biased distribution which satisfies the physics embodied in the constraints.

\subsection{Size and velocity estimation using the maximum entropy method}

The maximum entropy method can be used to determine the PDF of bubble pierced length, $y$, bubble diameter, $D$, and bubble velocity, $u_{b}$. The problem to be solved for the PDF of bubble pierced length 


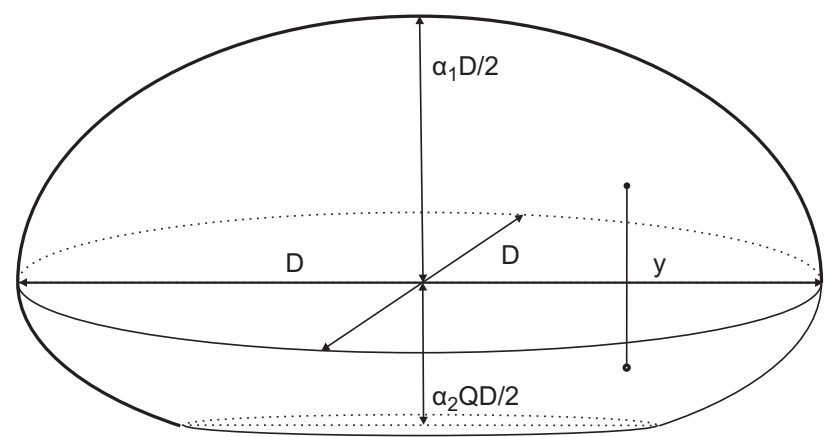

Fig. 1. Schematic of the truncated oblate ellipsoidal bubble.

estimation is

$$
\begin{aligned}
\max _{P(y)} & \int_{y \in \Delta}-P(y) \ln (P(y)) d y \\
\text { s.t } & \int_{y \in \Delta} y^{i} P(y) d y=\left\langle y^{i}\right\rangle, \quad i=1,2, \ldots, n \\
& \int_{y \in \Delta} P(y) d y=1
\end{aligned}
$$

The same formulation can be applied to obtain the PDF of the bubble velocity. The raw sample moments $\left\langle y^{i}\right\rangle$ and $\left\langle u_{b}^{i}\right\rangle$ are directly obtained from experimental measurements of $y$ and $u_{b}$.

A similar procedure could be used to calculate the PDF of the bubble diameter. However, the bubble diameter is a geometric parameter that cannot be measured experimentally and therefore, the corresponding raw sample moments $\left\langle D_{i}\right\rangle$ that are needed for the calculation, according to Eq. (2), are not directly available, and have to be estimated from the pierced lengths that are directly measured.

\subsection{Estimation of the probability distribution of bubble diameter from pierced length raw moments}

This section explains how to estimate the raw moments of the bubble diameter distribution using the sample raw moments of the pierced length distribution. The bubble geometry (Fig. 1) considered in the following formulation will be the most general one: a truncated oblate spheroid (an ellipsoid having two equal principal axis). This geometry has axial symmetry, thus it is independent of the azimuthal angle.

The pierced length PDF can be obtained assuming that bubbles will rise randomly distributed in a horizontal circular surface containing the probe (Werther, 1974b). This condition does not imply that the bubbles need to be uniformly distributed in the bed surface, but they just need to be homogeneously distributed locally, in the sense that the probability of the center of the bubble to cross this control surface is the same for any point of the surface. Furthermore, it was found by Santana et al. (2006) that considering the general case where the bubble velocity vector forms a certain angle of attack with the vertical direction, the errors in the angle of attack produce a negligible effect on the PDF estimation. Therefore, the ascending velocity will be considered vertical in the following formulation. Provided the bubble touches the probe and taking into account that pierced lengths smaller than the distance between probes ( $s$ ) cannot be measured, the maximum distance between the probe tip and the bubble symmetry axis that allows the bubble to be measured is, as shown in Fig. 2,

$$
r_{\max , s}= \begin{cases}\sqrt{\left(\frac{D}{2}\right)^{2}-\left(\frac{s-\alpha_{2} Q \frac{D}{2}}{\alpha_{1}}\right)^{2}}, & s>\left(\alpha_{1}+\alpha_{2}\right) \frac{D}{2} Q \\ \sqrt{\left(\frac{D}{2}\right)^{2}-\left(\frac{s}{\alpha_{1}+\alpha_{2}}\right)^{2}}, & s<\left(\alpha_{1}+\alpha_{2}\right) \frac{D}{2} Q\end{cases}
$$

Subindex $s$ in $r_{\max , s}$ indicates that this is the maximum distance between the probe tip and the bubble center for bubbles with pierced lengths larger than the distance between probes, $s$. This subindex will be used with this meaning hereafter.

Since a uniform distribution has been assumed for the distance between the probe and the bubble symmetry axis $r$, its cumulative distribution function is given by

$F_{S}\left(r \mid D, \alpha_{1}, \alpha_{2}, Q\right)=\frac{r^{2}}{r_{\max , S}^{2}}, \quad r \in\left(0, r_{\max , s}\right)$

Deriving this equation, the PDF of the distance $r$ between the probe and the bubble center for a given bubble diameter $D$ is obtained:

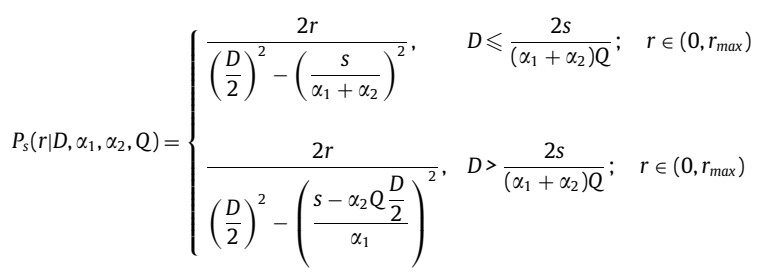

The pierced length measured by the probe is the distance between the points where the probe intersect the bubble surface in its ascension, and is given by (see Fig. 2)

$y= \begin{cases}\alpha_{1} \frac{D}{2} \sqrt{1-\left(\frac{2 r}{D}\right)^{2}}+\alpha_{2} Q \frac{D}{2}, & 0 \leqslant 2 r \leqslant D \sqrt{1-Q^{2}} \\ \left(\alpha_{1}+\alpha_{2}\right) \frac{D}{2} \sqrt{1-\left(\frac{2 r}{D}\right)^{2}}, & D \sqrt{1-Q^{2}} \leqslant 2 r \leqslant D\end{cases}$

Then the following statistical property can be used:

$P_{s}\left(y \mid D, \alpha_{1}, \alpha_{2}, Q\right)=P_{s}\left(r \mid D, \alpha_{1}, \alpha_{2}, Q\right)\left|\frac{d r}{d y}\right|$

where

$\left|\frac{d y}{d r}\right|= \begin{cases}\frac{\alpha_{1}^{2} r}{y-\alpha_{2} Q \frac{D}{2}}, & 0 \leqslant 2 r \leqslant D \sqrt{1-Q^{2}} \\ \frac{\left(\alpha_{1}+\alpha_{2}\right)^{2} r}{y}, & D \sqrt{1-Q^{2}} \leqslant 2 r \leqslant D\end{cases}$ 


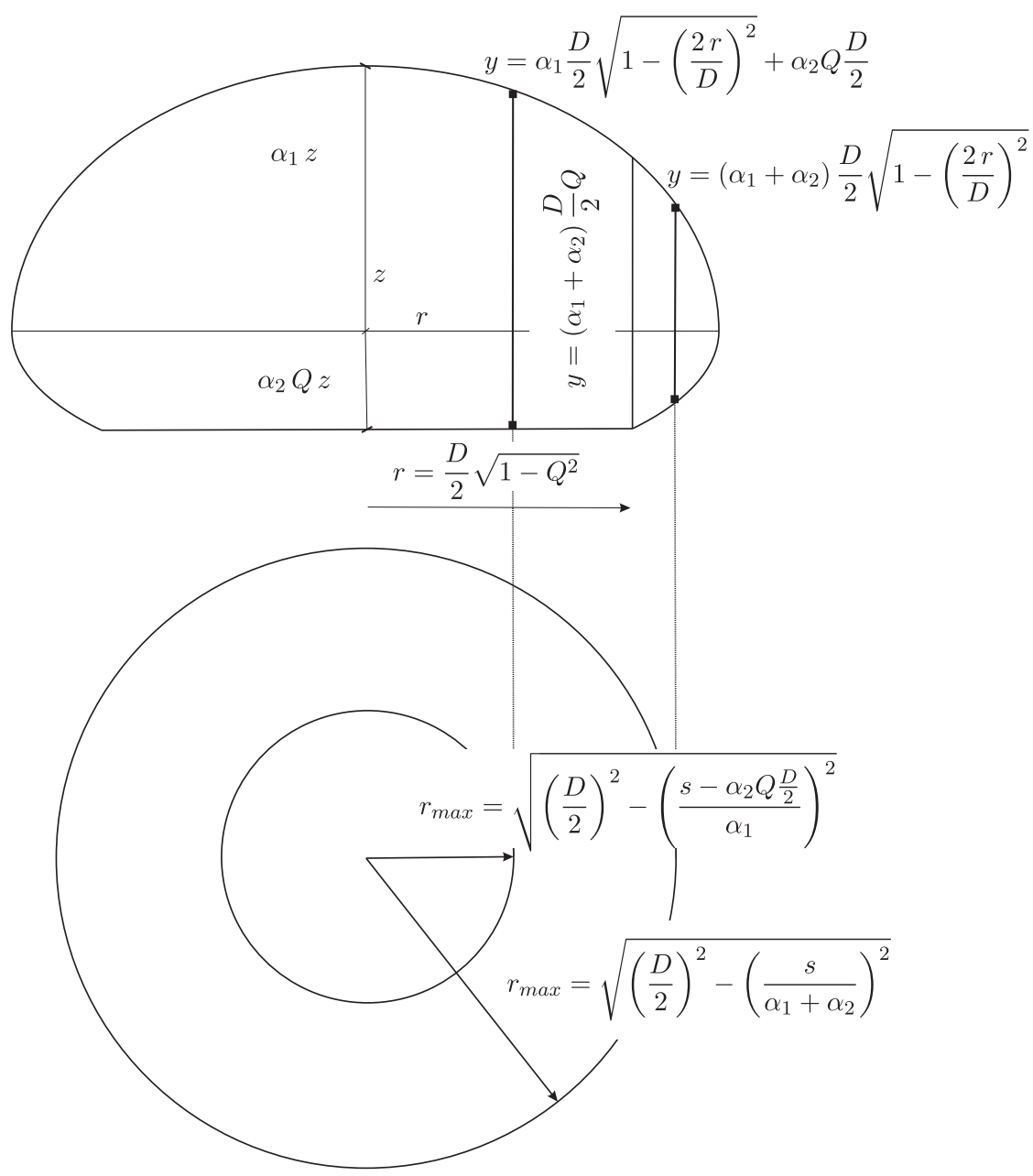

Fig. 2. Vertical section of the truncated ellipsoidal bubble.

Then if the bubble diameter is known, the PDF of pierced lengths can be obtained as follows:

$P_{s}\left(y \mid D, \alpha_{1}, \alpha_{2}, Q\right)=\left\{\begin{array}{l}D \leqslant \frac{2 s}{\left(\alpha_{1}+\alpha_{2}\right) Q}\left\{\begin{array}{l}\frac{2\left(y-\alpha_{2} Q \frac{D}{2}\right)}{\alpha_{1}^{2}\left[\left(\frac{D}{2}\right)^{2}-\left(\frac{\left.s-\alpha_{2} Q \frac{D}{2}\right)^{2}}{\alpha_{1}}\right)^{2}\right]} \\ D>\frac{2 s}{\left(\alpha_{1}+\alpha_{2}\right) Q}\left\{\begin{array}{l}\frac{2\left(y-\alpha_{2} Q \frac{D}{2}\right)}{\alpha_{1}^{2}\left[\left(\frac{D}{2}\right)^{2}-\left(\frac{s}{\alpha_{1}+\alpha_{2}}\right)^{2}\right]} \\ \frac{\left(\alpha_{1}+\alpha_{2} Q\right) \frac{D}{2} \geqslant y \geqslant\left(\alpha_{1}+\alpha_{2}\right)}{2} \frac{D}{2} \\ \left(\alpha_{1}+\alpha_{2}\right)^{2}\left[\left(\frac{D}{2}\right)^{2}-\left(\frac{s}{\alpha_{1}+\alpha_{2}}\right)^{2}\right]\end{array}\right. \\ \left(\alpha_{1}+\alpha_{2}\right) Q \frac{D}{2} \geqslant y \geqslant s\end{array}\right.\end{array}\right.$
The raw moment of order $i$ of the pierced length $y$ can be calculated applying the total probability theorem

$$
\begin{aligned}
\left\langle y^{i} \mid \alpha_{1}, \alpha_{2}, Q\right\rangle & =\int_{s}^{\infty} y^{i} P_{s}\left(y \mid \alpha_{1}, \alpha_{2}, Q\right) d y \\
& =\int_{s}^{\infty} y^{i} \int_{2 y /\left(\alpha_{1}+\alpha_{2} Q\right)}^{\infty} P_{s}\left(y \mid D, \alpha_{1}, \alpha_{2}, Q\right) P_{s}\left(D \mid \alpha_{1}, \alpha_{2}, Q\right) d D d y
\end{aligned}
$$

The lower limit of the last integral in Eq. (13) is the minimum diameter, $D_{\min }$, that is possible to obtain provided the minimum measurable pierced length is $s$. Inverting the integral limits and introducing Eq. (12) in the above expression the following equation is obtained:

$$
\begin{aligned}
& \left\langle y^{i} \mid \alpha_{1}, \alpha_{2}, Q\right\rangle=\int_{2 s /\left(\alpha_{1}+\alpha_{2} Q\right)}^{2 s /\left(\alpha_{1}+\alpha_{2}\right) Q} \int_{s}^{\left(\alpha_{1}+\alpha_{2} Q\right) D / 2} \\
& \times \frac{y^{i} 2\left(y-\alpha_{2} Q \frac{D}{2}\right)}{\alpha_{1}^{2}\left[\left(\frac{D}{2}\right)^{2}-\left(\frac{s-\alpha_{2} Q \frac{D}{2}}{\alpha_{1}}\right)^{2}\right]} d y P_{s}\left(D \mid \alpha_{1}, \alpha_{2}, Q\right) d D \\
& \times \frac{\int_{2 s /\left(\alpha_{1}+\alpha_{2}\right) Q}^{\left(\alpha_{1}+\alpha_{2}\right) Q D / 2} \int_{s} y^{i+1}}{\left(\alpha_{1}+\alpha_{2}\right)^{2}\left[\left(\frac{D}{2}\right)^{2}-\left(\frac{s}{\alpha_{1}+\alpha_{2}}\right)^{2}\right]} d y P_{s}\left(D \mid \alpha_{1}, \alpha_{2}, Q\right) d D
\end{aligned}
$$




$$
\begin{gathered}
+\int_{2 s /\left(\alpha_{1}+\alpha_{2}\right) Q}^{\infty} \int_{\left(\alpha_{1}+\alpha_{2}\right) Q D / 2}^{\left(\alpha_{1}+\alpha_{2} Q\right) D / 2} \\
\times \frac{y^{i} 2\left(y-\alpha_{2} Q \frac{D}{2}\right)}{\alpha_{1}^{2}\left[\left(\frac{D}{2}\right)^{2}-\left(\frac{s}{\alpha_{1}+\alpha_{2}}\right)^{2}\right]} d y \\
\times P_{S}\left(D \mid \alpha_{1}, \alpha_{2}, Q\right) d D
\end{gathered}
$$

Considering the integration limits of the first term in Eq. (14) and the properties of the PDF this term can be neglected in the calculation of the integral (in this case its contribution is less than 1\%) obtaining

$$
\begin{aligned}
& \frac{i+2}{2}\left\langle y^{i} \mid \alpha_{1}, \alpha_{2}, Q\right\rangle \\
& =\int_{2 s /\left(\alpha_{1}+\alpha_{2} Q\right)}^{\infty} \frac{\left(\frac{D}{2}\right)^{i+2} S_{i}-s^{i+2}}{\left(\alpha 1+\alpha_{2}\right)^{2}\left(\left(\frac{D}{2}\right)^{2}-\left(\frac{s}{\alpha_{1}+\alpha_{2}}\right)^{2}\right)} P_{S}\left(D \mid \alpha_{1}, \alpha_{2}, Q\right) d D
\end{aligned}
$$

where

$S_{i}=\frac{\left(\alpha_{1}+\alpha_{2}\right)^{i+2}}{(i+1) \alpha_{1}^{2}}\left[Q^{i+2} \alpha_{2}\left(\alpha_{2}-i \alpha_{1}\right)+\frac{\left(\alpha_{1}+Q \alpha_{2}\right)^{i+1}}{\left(\alpha_{1}+\alpha_{2}\right)^{i}}\left(\alpha_{1}(i+1)-\alpha_{2} Q\right)\right]$

In the estimation of the distribution $P_{S}(D)$ it was considered that only pierced lengths larger than the distance between probes $(y>s)$ can be measured by the probes. In other words, $P_{S}(D)$ in Eq. (15) is the diameter distribution of the bubbles that get immersed in both probes in its ascension. Then of applying Bayes theorem to obtain the PDF of $D, P(D)$,

$P\left(D_{s} \mid D\right)=P\left(D \mid D_{s}\right) \frac{P_{s}(D)}{P(D)}$

where

$P\left(D \mid D_{S}\right)=1$

$P\left(D_{s} \mid D\right)=\frac{D^{2}-\left(\frac{2 s}{\alpha_{1}+\alpha_{2}}\right)^{2}}{D^{2}}$

Hence the following expression is obtained for $P(D)$ :

$\frac{i+2}{2}\left\langle y^{i} \mid \alpha_{1}, \alpha_{2}, Q\right\rangle=\int_{2 s /\left(\alpha_{1}+\alpha_{2} Q\right)}^{\infty} \frac{\left(\frac{D}{2}\right)^{i+2} S_{i}-s^{i+2}}{\left(\alpha_{1}+\alpha_{2}\right)^{2}\left(\frac{D}{2}\right)^{2}} P\left(D \mid \alpha_{1}, \alpha_{2}, Q\right) d D$

where $S_{i}$ is given in Eq. (16). Then, applying the statistical property $P(D) d D=P\left(D_{v}\right) d D v$, an expression to estimate $P\left(D_{v}\right)$ can be derived from Eq. (19), $D_{v}$ being the volume equivalent diameter which is the diameter of a sphere having the same volume as the bubble:

$$
\begin{aligned}
\frac{i+2}{2}\left\langle y^{i} \mid \alpha_{1}, \alpha_{2}, Q\right\rangle= & \int_{\left(2 s /\left(\alpha_{1}+\alpha_{2} Q\right)\right) \rho^{1 / 3}}^{\infty} \frac{\left(\frac{D_{v}}{2}\right)^{i+2} \rho^{-(i+2) / 3} S_{i}-s^{i+2}}{\left(\alpha_{1}+\alpha_{2}\right)^{2}\left(\frac{D_{v}}{2}\right)^{2} \rho^{-2 / 3}} \\
& \times P\left(D_{v} \mid \alpha_{1}, \alpha_{2}, Q\right) d D_{v}
\end{aligned}
$$

where $\rho$ is the ratio of the volume of the bubble, $V$, to the volume of a sphere of the same diameter, $V_{b}$. And calculating the volume of the bubble considering the geometry shown in Fig. 1 it can be seen that

$\rho=\frac{V}{V_{b}}=\left(\frac{D_{v}}{D}\right)^{3}=\frac{\alpha_{2} Q\left(3-Q^{2}\right)+2 \alpha_{1}}{4}$
Then the raw moments of the pierced length (left-hand side of Eq. (20)) can be related with an expectation function of the bubble diameter (right-hand side of Eq. (20)). This relation provides the constrains in the system equations (2), needed to calculate the diameter size distribution. Finally, once the raw moments are estimated from the sample moments, the maximum entropy distribution subject to $n$ constrains takes the following form (Sellens and Brzustowski, 1985):

$P\left(D_{v}\right)=\exp \left[-\lambda_{0}-\sum_{i=1}^{n} \lambda_{i} \frac{\left(\frac{D_{v}}{2}\right)^{i+2} \rho^{-(i+2) / 3} S_{i}-s^{i+2}}{\left(\alpha_{1}+\alpha_{2}\right)^{2}\left(\frac{D_{v}}{2}\right)^{2} \rho^{-2 / 3}}\right]$

where $\lambda_{i}$ is the Lagrange multiplier for the $i$ th constraint and $\lambda_{0}$ is a normalization constant so the integral of the PDF is 1. Expanding the last equation, the following expression is obtained:

$$
\begin{aligned}
P\left(D_{v}\right)= & \exp \left[-\lambda_{0}-\sum_{i=1}^{n} \lambda_{i} \frac{\left(\frac{D_{v}}{2}\right)^{i} \rho^{-i / 3} S_{i}}{\left(\alpha_{1}+\alpha_{2}\right)^{2}}\right] \\
& \times \exp \left[\sum_{i=1}^{n} \lambda_{i} \frac{s^{i+2}}{\left(\alpha_{1}+\alpha_{2}\right)^{2}\left(\frac{D_{v}}{2}\right)^{2} \rho^{-2 / 3}}\right]
\end{aligned}
$$

The first term in Eq. (23) takes into account the diameter distribution of bubbles touching the probe, whereas the second term corrects the underestimation of the smaller bubbles because of the lower probability of being engulfed by the probe. For bubbles of size larger than the probe spacing the second term vanishes because the probability of being engulfed by two or one tip is nearly the same.

Santana et al. (2006) applied this method without considering the distance between probes $s$ in the calculation of $P(y)$. In that case, the resulting equation relates directly the raw moments of the diameter with the pierced length and consequently different moments of the bubble diameter can be obtained analytically from measured pierced lengths $\left(f_{i}=D^{i}\right.$ in their work). Eq. (19) shows that this does not happen when the distance $s$ is taken into account, since the function $f_{i}$ is a more complex function of $D$. Therefore the obtained $P(D)$ must be numerically integrated in order to obtain the different moments of the bubble diameter.

Further details in the numerical implementation of this method can be found in Rockinger and Jondeau (2002) and Santana et al. (2006).

\subsubsection{Shape factor}

To calculate the bubble size distribution the shape factor of the bubbles needs be known. Luther et al. (2004) measured the shape factor of bubbles in gas-liquid systems using a four-point probe. The experimental measurement of the bubble shape factor in threedimensional fluidized beds is rather complicated. In this work the bubble shape will be estimated using the correlation proposed by Werther (1976) for the shape factor, $\rho$, which is the ratio of the volume of the bubble to the volume of a sphere of the same diameter:

$\rho=\left(1-0.3 \exp \left[-8\left(U-U_{m f}\right)\right]\right) \exp (-\phi z)$

$\phi=7.2\left(U-U_{m f}\right) \exp \left[-4.1\left(U-U_{m f}\right)\right]$

where $z$ and $\left(U-U_{m f}\right)$ are in $\mathrm{m}$ and $\mathrm{m} / \mathrm{s}$, respectively. Werther (1974a) showed how an ellipsoidal bubble shape may be easily assimilated to another one with a shape similar to that of a spherical cap bubble, without altering the maximum horizontal and vertical dimension of the bubble, nor its volume or the distribution of pierced lengths. Therefore, the parameters $\alpha_{1}$ and $\alpha_{2}$ have been chosen equal to 1 . The geometry factor $Q$ in Fig. 1 can be calculated from $\rho$ using Eq. (21). 
a



b

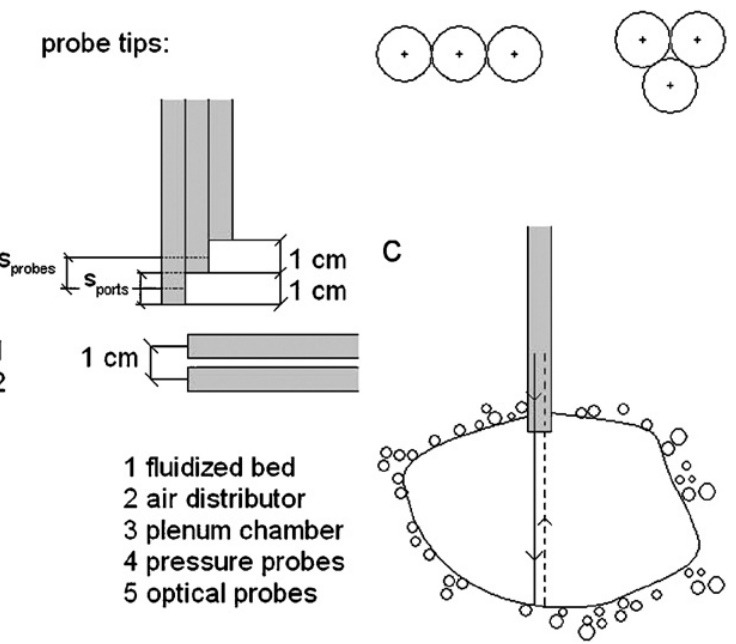

Fig. 3. (a) Experimental set-up. (b) Pressure probes configuration. (c) Optical probe light reflected by the particle phase.

\section{Experiments}

Different experiments were carried out in a bubbling fluidized bed of $0.193 \mathrm{~m}$ ID and $0.8 \mathrm{~m}$ height. The fluidized bed column was filled with Geldart B silica sand particles with a mean diameter of $680 \mu \mathrm{m}$ and a density of $2632.5 \mathrm{~kg} / \mathrm{m}^{3}$. The settled bed height was $22 \mathrm{~cm}$. The column had a perforated plate to distribute the air with 90 holes of $2 \mathrm{~mm}$ diameter laid out in hexagonal pitch of $15 \mathrm{~mm}$. The total open area ratio of the distributor was $1 \%$. The minimum fluidization velocity of this fluidized bed was $U_{m f}=0.4 \mathrm{~m} / \mathrm{s}$ and the distributor to bed pressure drop ratio for this velocity was $\Delta P_{d} / \Delta P_{b}=0.48$.

Pressure and optical probe measurements had a duration of $10 \mathrm{~min}$ and the sample frequency was $500 \mathrm{~Hz}$. The data were recorded with a 12 bits data acquisition board (ICP DAS PCI-1802H) assembled in a PC. Optical probes developed for this study are based on backscattering principle.

\subsection{Pressure probes}

Gauge pressure measurements were carried out using piezoresistive differential pressure transducers Omega PX $291\left(0-5\right.$ in $\left.\mathrm{H}_{2} \mathrm{O}\right)$ with a $1 \% \mathrm{FS}$ accuracy. The high pressure port was connected to a probe immersed in the bed and the low pressure port was exposed to the atmosphere. The pressure measurement accuracy was approximately \pm 12 Pa.

Three pressure probes of $4 \mathrm{~mm}$ external diameter were located at the bed axis opposed to the flow direction with the probe tip at a distance of 12,13 and $14 \mathrm{~cm}$ from the distributor. Pressure probes had to be positioned in the axis direction to enhance the transmission of the pressure signals. No mesh was installed at the pressure probe tip since it dampens the signal. For this reason a probe diameter of $4 \mathrm{~mm}$ had to be chosen to prevent particles to clog the probe. The vertical position of the probe prevented from particles getting to the transducer. The three probes were positioned tangentially (Fig. 3(b) left) because although a triangular configuration (Fig. 3(b) right) presents a lower eccentricity between probes, it hinders the reception of the pressure signal due to the presence of the other two probes.

\subsection{Optical probes}

In-house developed optical probes were also used in the experiments. Two probes were placed at the bed axis at a distance of 10

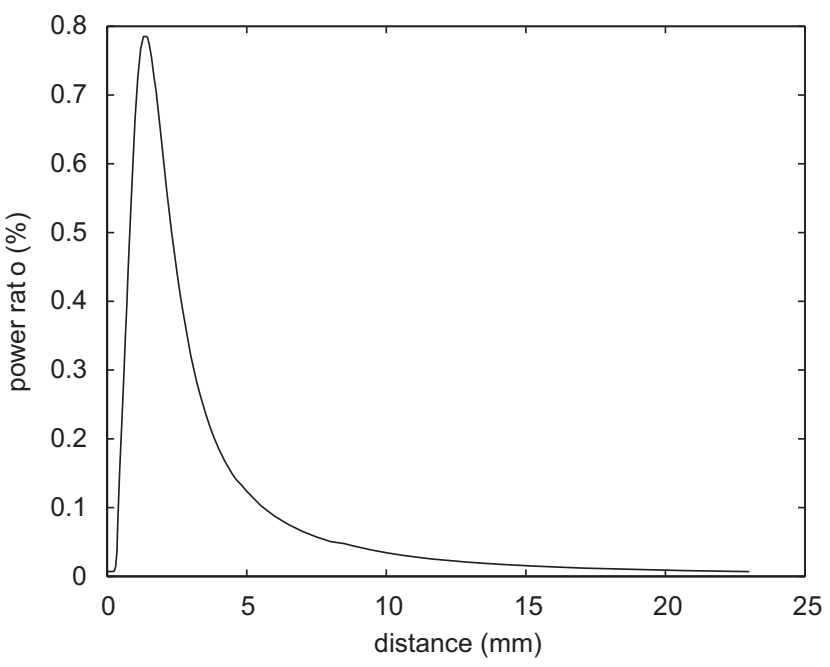

Fig. 4. Reflective curve of the optical fiber probe.

and $11 \mathrm{~cm}$ from the distributor. Optical probes were located $1 \mathrm{~cm}$ below the pressure probe bundle to avoid the interference in the optical probe light. A schematic of the set up is shown in Fig. 3(a). The OFP were made of two standard step-index plastic optical fibers (emitter and receiver fibers) embedded in a metallic coil of $3 \mathrm{~mm}$ external diameter for avoiding bending influences. The fibers had a diameter of $1 \mathrm{~mm}$ with $0.22 \mathrm{~dB} / \mathrm{m}$ attenuation and $0.47 \mathrm{NA}$. The propagation delay constant of the fiber was $5 \mathrm{~ns} / \mathrm{m}$ and the whole probe had a length of about $1 \mathrm{~m}$. The diameter of the fiber was larger than the particle size and smaller than the bubble characteristic size, thus these fibers are suitable for measuring both voidage and bubble parameters (Liu and Clark, 1995). The emitter fiber was illuminated by a $650 \mathrm{~nm}$ laserdiode with a maximum power of $10 \mathrm{~mW}$ (Roithner $56510 \mathrm{mg}$ ). A phototransistor was used at the reception as part of a transimpedance amplifier, giving an output voltage proportional to the output optical power. The laserdiode and the phototransistor were encapsulated in ST connectors. A 50/50 passive splitter is used for splitting the optical power into the emitter fibers of both probes. The optical probes are suitable for an operating temperature 


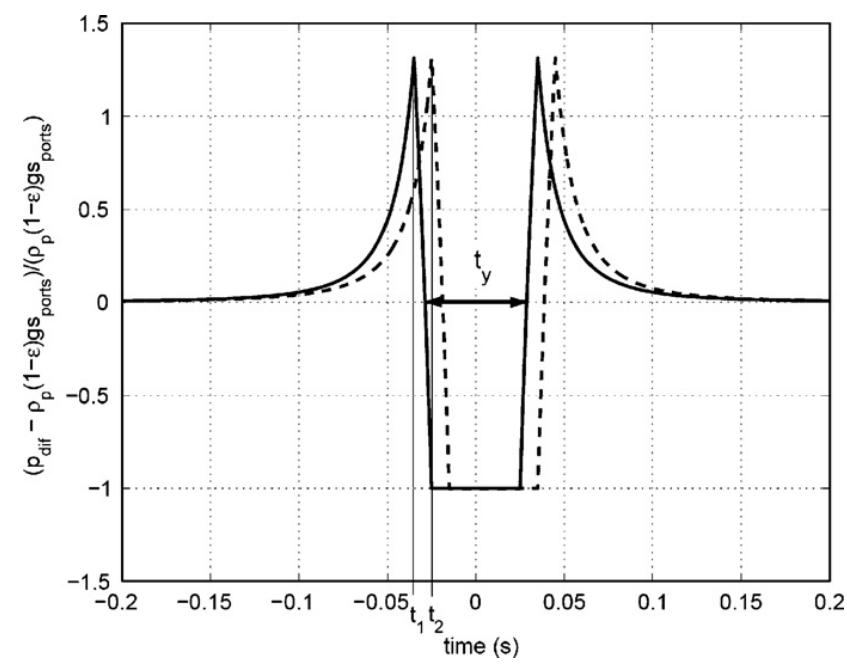

Fig. 5. Normalized differential pressure field around a rising bubble. Solid line: $p_{\text {dif } 1}$, dashed line: $p_{\text {dif2 }}$.

from -40 to $85^{\circ} \mathrm{C}$. Fig. 4 presents the experimental reflective curve for the developed optical probe. The power ratio is expressed in percentage and it is the power of the light captured by the receiving fiber divided by that delivered by the emitter fiber. This curve has been calculated positioning a white surface at increasing distances from the probe tip. From Fig. 4, it is possible to specify limits of the penetration depth. For a distance of $5 \mathrm{~mm}$ the signal intensity falls about a $10 \%$ its maximum value, hence this may be considered the outer limit of the measuring volume of the probe (Liu et al., 2003). The optical probes have to be placed perpendicular to the flow to preclude the light reflected at the bottom of the bubble from affecting the determination of the time instant the probe leaves the bubble (see Fig. 3(c)), what would make more difficult to distinguish between bubble and emulsion phase in the subsequent signal processing.

\section{Signal processing}

\subsection{Pressure signal}

The differential pressure signal has been widely proposed as a useful method to study bubble properties in fluidized beds (Dent et al., 1989; Sitnai, 1982; Venkata Ramayya et al., 1996). Several authors (Littman and Homolka, 1973; Sitnai, 1982; Venkata Ramayya et al., 1996) have proved that Davidson's model (Davidson and Harrison, 1963) represents well the pressure field around a rising bubble in a fluidized bed. Sitnai (1982) also noted that knowledge of the precise details of the pressure field is not essential for the determination of the main bubble parameters. Indeed the dominant features of the real pressure field are a negligible pressure gradient inside the bubble and high recovery gradients at the bubble nose and tail. Fig. 5 shows the pressure distribution around a bubble resulting from Davidson's model. The normalized differential pressure predicted with this model is plotted against time. The solid line in Fig. 5 is the differential pressure $p_{\text {dif } 1}$ obtained subtracting the gauge pressure at the middle port $p_{2}$, from the gauge pressure $p_{1}$ at the lower pressure port. The dashed line is the differential pressure $p_{\text {dif } 2}$ obtained subtracting the gauge pressure at the upper port, $p_{3}$, from $p_{2}$. Care must be taken to keep the same dead volume in the three pressure lines (Clark and Atkinson, 1988), otherwise signals are generally spurious and as much dependent on global fluctuations in the bed as on local differential pressures.
Other authors (Sitnai, 1982; Dent et al., 1989; Venkata Ramayya et al., 1996) installed two pairs of pressure probes (one above the other) and connected each pair to a differential pressure transducer. On the contrary, for the configuration chosen here, the distance between probes $s$ and the distance between ports, $s_{\text {ports }}$, are necessarily equal (see Fig. 3(a)). Nevertheless, this configuration has the advantage of requiring only three pressure probes to be immersed in the bed.

The bubble ascending velocity, $u_{b}$, and the bubble pierced length, $y$, can be determined from differential pressure measurements. In Fig. 5 it can be observed that at $t_{1}$ the bubble nose reaches the lower pressure port while at $t_{2}$, the bubble reaches the upper one. Hence bubble velocity can be calculated as

$u_{b}=\frac{s}{t_{u}}$

where $t_{u}$ is the time lag $t_{2}-t_{1}$.

The time period between the two instants where the pressure gradient curve crosses the reference line (labeled $t_{y}$ in Fig. 5) was approximated by Sitnai (1982) as equivalent to the ratio of bubble pierced length to its rise velocity $\left(y / u_{b}\right)$. Therefore, the bubble pierced length may be computed as

$y=u_{b} \cdot t_{y}$

It can be easily deduced from Eq. (26) that the relative error of the calculated velocity is mainly given by

$e_{u_{b}}=\frac{\Delta u_{b}}{u_{b}}=\frac{\Delta t}{t_{u}}=\frac{u_{b}}{s} \cdot \Delta t$

where $\Delta t=0.002 \mathrm{~s}$ is the time between samples.

The relative error of $y$ can be deduced from Eq. (27):

$e_{y}=\Delta t\left(\frac{1}{t_{u}}+\frac{1}{t_{y}}\right)=\frac{u_{b}}{s} \cdot \Delta t+\frac{u_{b}}{y} \Delta t$

This error analysis shows that a higher distance between pressure probes minimizes the error in the calculation of bubble velocity. However, if this distance is too high it may happen that the bubble does not cross both pressure probes when it rises. Therefore, there must be a compromise between those parameters. In the present work a separation between probes of $s=1 \mathrm{~cm}$ has been chosen. Eq. (29) also shows that if $y<s$ the second term in the equation dominates over the first one; the relative error of $y, e_{y}$, increases when $y$ decreases. For this reason pierced length measurements smaller than $s$ have not been considered reliable.

An example of the differential pressure measured in the bed is shown in Fig. 6(a). The bubble velocity is determined from the time delay between the two differential pressure signals. However, Werther (1974b) showed that the instantaneous velocity of a bubble is a stochastic quantity, thus it is convenient to divide the signal in time portions and treat each bubble independently. In this way, the velocity of the demarcated bubble is obtained by applying correlation techniques only over the length of the signal corresponding to an individual bubble (Ghadiri et al., 1988).

The first criterion that must be fulfilled in order to detect a bubble presence is that $p_{\text {dif } 1}$ and $p_{\text {dif } 2}$ fall below their mean value $\left(\rho_{p} g(1-\varepsilon) s_{\text {ports }}\right)$ and approach zero. A further criterion has been used to reject bubbles rising with an horizontal eccentricity greater than the bubble radius. In this case the record differential pressure falls half of its average value (Dent et al., 1989; Sitnai, 1982). Bubbles detected following these criteria are marked with an arrow in Fig. 6(a).

Once the bubble is detected, bubble velocity is obtained from the cross correlation of the differential pressure signals (Sitnai, 1982) as shown in Fig. 7(b). The cross correlation is computed in the time interval spanning from the first maximum of $p_{\text {dif } 1}$ to the second 
a

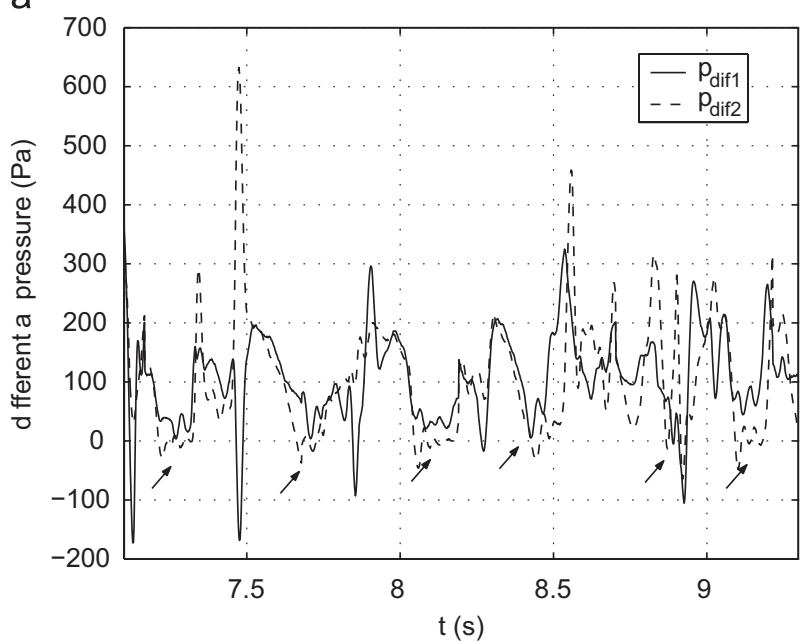

b



Fig. 6. (a) Differential pressure signals $p_{\text {dif } 1}$ and $p_{\text {dif } 2}$. (b) Optical probes signals $o p_{1}$ and $o p_{2}$. Bubble passages are marked with arrows.

maximum of $p_{\text {dif } 2}$ (Fig. 7(a)). The maximum of the cross correlation occurs for a time $s / u_{b}$. Once the bubble velocity, $u_{b}$, has been obtained it can be used to determine the bubble pierced length, $y$, which also depends on the time interval $t_{y}$. This time interval has been obtained from the raw signal (Venkata Ramayya et al., 1996) as shown in Fig. 7(a). $p_{\text {dif } 1}$ has been chosen to perform this calculation since the bubble distortion caused by the probe is weaker at this moment.

\subsection{Optical probe signal}

A similar procedure is used to calculate bubble characteristics from optical signals. Fig. 6(b) shows an example of the raw signal measured by optical probes $o p_{1}$ and $o p_{2}$. When a bubble passes in front of the probe the reflected light is lower than the reflected light by the emulsion phase (high concentration of particles) and the voltage falls rapidly.

The criterion chosen to detect bubbles from the optical signal is a threshold voltage (bubble detection threshold). When the signalfalls below this value, it is considered a bubble passage is occurring. a

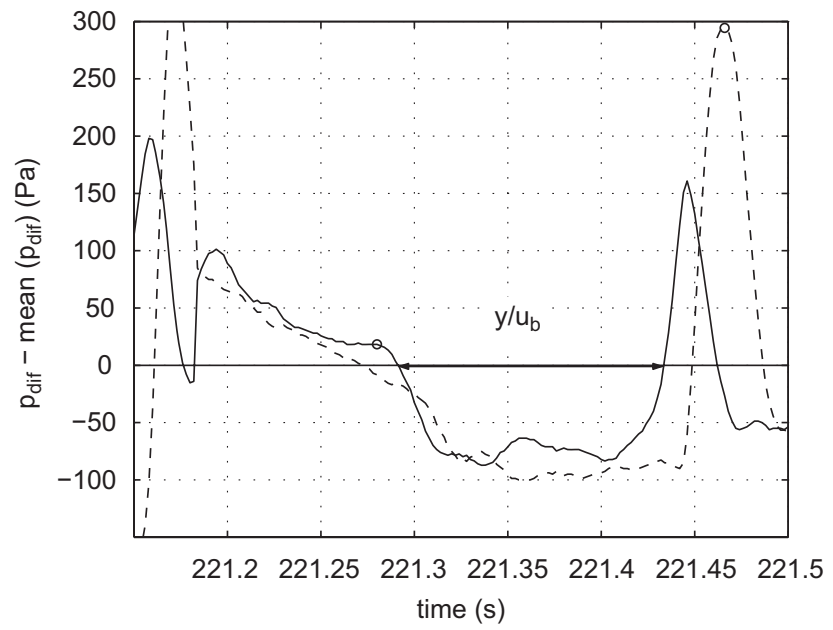

b

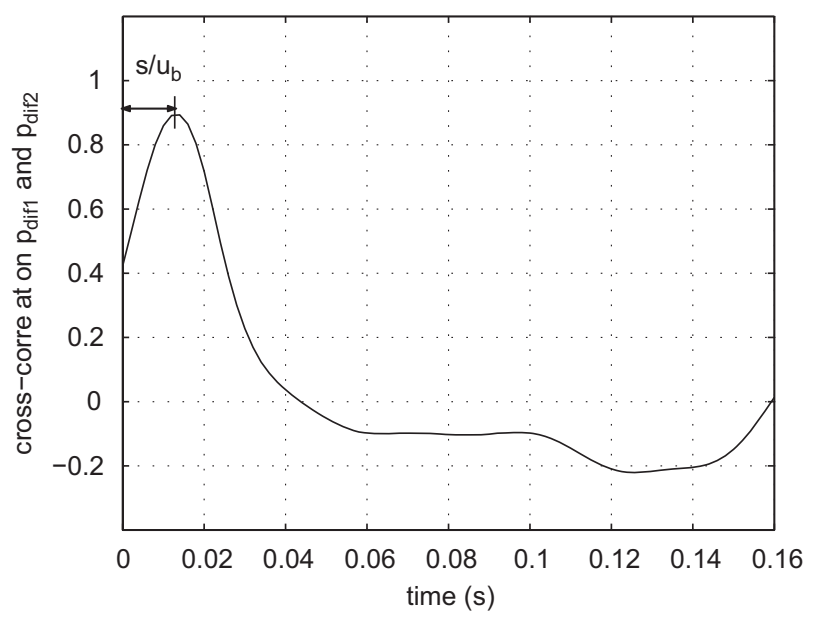

Fig. 7. (a) Differential pressure signals $p_{\text {dif } 1}$ and $p_{\text {dif } 2}$. (b) Cross correlation of the differential pressure signals. The cross correlation was computed for the time interval marked with circles.

This threshold can be determined by plotting the histogram of the output voltage from the optical probes (Schweitzer et al., 2001). Fig. 8 shows an example. It exhibits a peak corresponding to emulsion-phase responses at voltage around $2 \mathrm{~V}$ and a tail at lower voltages corresponding to gas bubbles. The bubble detection threshold has been defined as the voltage where the histogram tail begins, i.e. where the slope of the histogram becomes nearly zero. Bubbles detected following this criterion are marked with an arrow in Fig. 6(b). When two bubbles are very close it is subjective whether to consider them as one or two bubbles. The assignment of the bubble boundary location is also subjective due to the high void fraction region at the boundary, and the relatively large particle content inside the bubbles (Mainland and Welty, 1995). In the present work, the mean value of the signal has been considered an appropriate threshold to demarcate the bubble length (bubble length threshold), since bubble properties are not overly sensitive to this value, due to the fact that the signal level drops very fast when a bubble crosses the probe.

Signals $o p_{1}$ and $o p_{2}$ were correlated in order to calculate bubble velocity as explained for the pressure signals (Fig. 9(b)). The time interval where the cross correlation was computed was that part of 


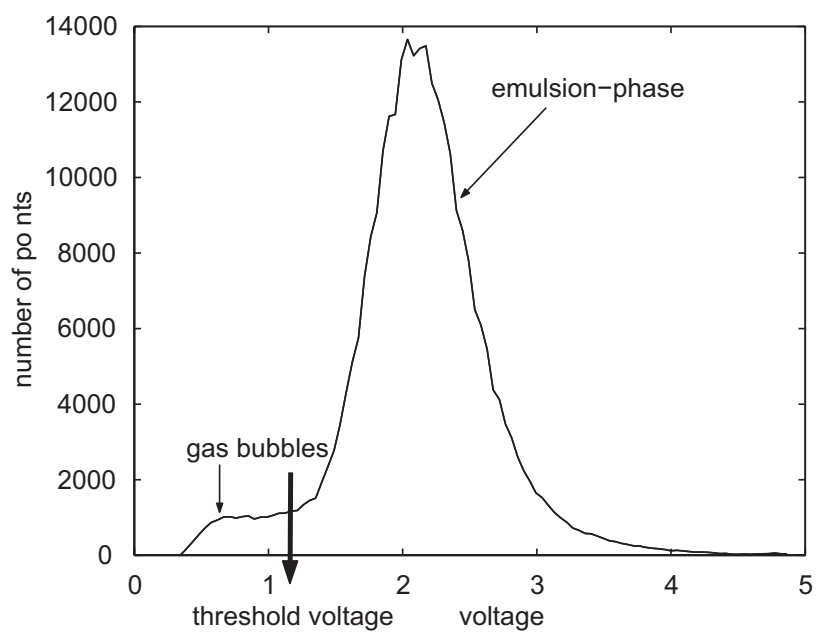

Fig. 8. Optical signal histogram.

the signal between the maxima found just before and after the bubble boundaries, where the signal begins to fall abruptly. The piercing time, $t_{y}$, is the time during which the probe is immersed into the bubble. It has been calculated from the lower probe signal as the time interval between the two instants where the signal crosses its mean value (see Fig. 9(a)).

There are bubbles that are detected by the pressure probes but not by the optical probes since they do not meet the detection criterion. This occurs because optical probes provide more local measurements and they do not detect a bubble unless the measuring volume is to some degree free of particles.

The processing of pressure and optical probe signals provides a sample of pierced lengths with their corresponding velocities. After that, the following criteria are applied to reject erroneous measurements: pierced length measurements smaller than the separation between probes $s$ are considered inaccurate, pierced lengths larger than the bed radius have been considered outliers and bubble velocity measurements higher than $1 \mathrm{~m} / \mathrm{s}$ have also been rejected.

\section{Results and discussion}

PDF of bubble pierced length, velocity and diameter were obtained using the method described above. Results from pressure and optical measurements are compared in Fig. 10. The mean and standard deviation of $y$ and $D_{v}$ are summarized in Table 1.

Fig. 10(a) shows that the PDF of pierced lengths obtained from pressure measurements exhibits a second peak around $y=8 \mathrm{~cm}$. This behavior is not observed in the PDF obtained from the optical measurements. This phenomenon can be attributed to the beginning of the coalescence phenomenon, since first, the pressure probes are located higher in the bed and second, it is not possible to distinguish between two bubbles coalescing and one single large bubble using pressure signals. PDF of bubble velocity (Fig. 10(b)) can be considered somehow a uniform distribution. Bubble diameter and bubble equivalent diameter distributions (Fig. 10(c) and 10(d)) show a mode value very similar in both pressure and optical measurements. Slight differences may be explained by the difference in the height position. Moreover it can be observed that the variance of the distribution obtained from the pressure measurements is higher than that of the distribution obtained using optical probes. This difference is
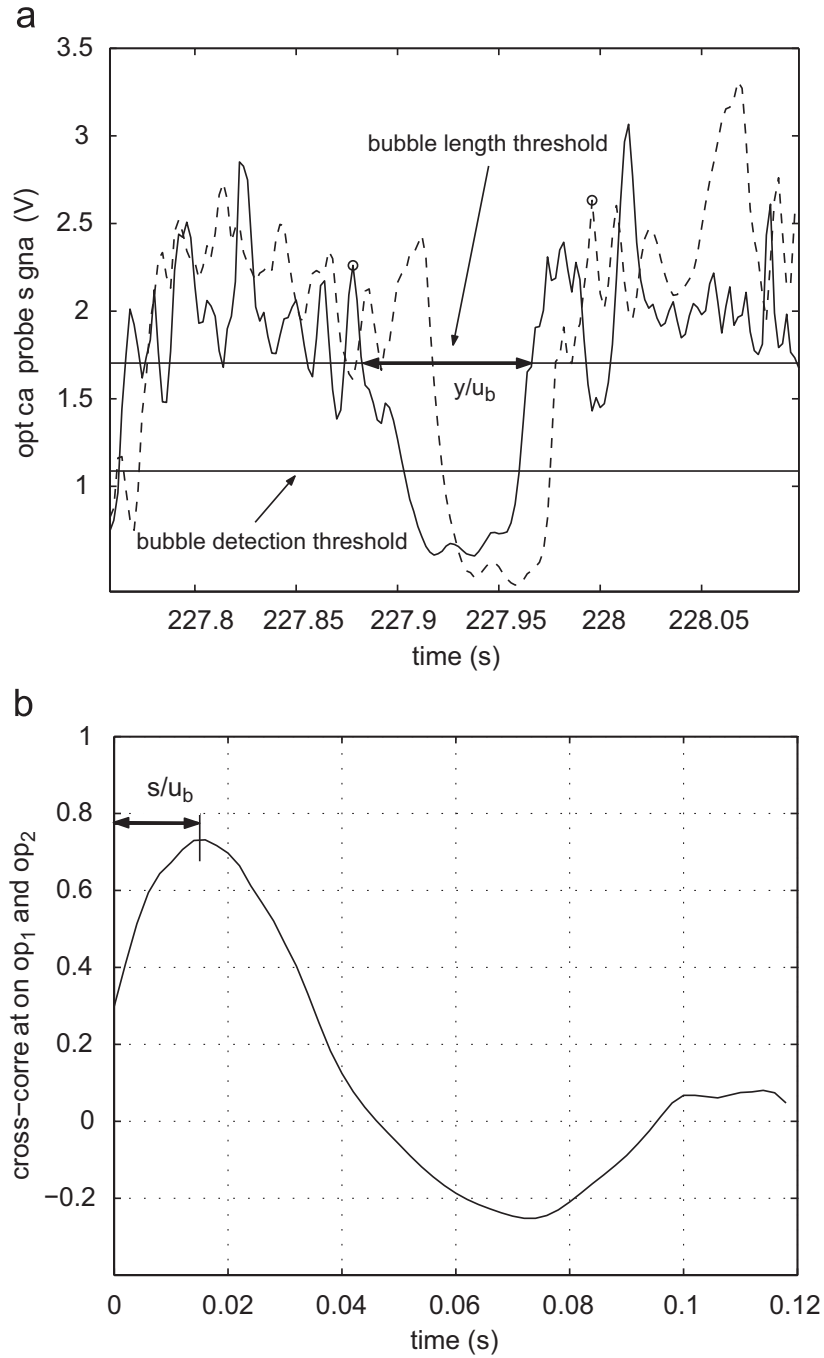

Fig. 9. (a) Optical probe signals $o p_{1}$ and $o p_{2}$. (b) Cross correlation of the optical probe signals. The cross correlation was computed in the time interval marked with circles.

probably due to the different position of the probes but also, to the fact that the pressure signals are influenced by other phenomena but the bubble passage whereas optical are very good phase detection probes.

Bubble characteristics were also measured at a lower height in the bed, using optical probes. It was not possible to obtain bubble parameters from pressure signals close to the distributor as the bubble detection was very difficult due to the small size of the bubbles.

Results at a height $z=5.5 \mathrm{~cm}$ above the distributor and a radial position $r / R=0.8$ are shown in Fig. 11. The mean and standard deviation of $y$ and $D_{v}$ are summarized in Table 1. Comparing Figs. 10 and 11 it is shown that as expected, the mean values of $y, D$ and $D_{v}$ are smaller for the lower height. The variance is also smaller. In this case, the mode of the distribution is greater than $s$. However, the mean value and the standard deviation are, as noted earlier, smaller. Observing the $\operatorname{PDF}(y)$ in Fig. 11(a) it can be concluded that just a small portion of the distribution is truncated. This is also the case for the $\operatorname{PDF}\left(D_{v}\right)$ (or $\left.\operatorname{PDF}(D)\right)$ for both cases $(z=10.5$ and $5.5 \mathrm{~cm})$. Observing 
a

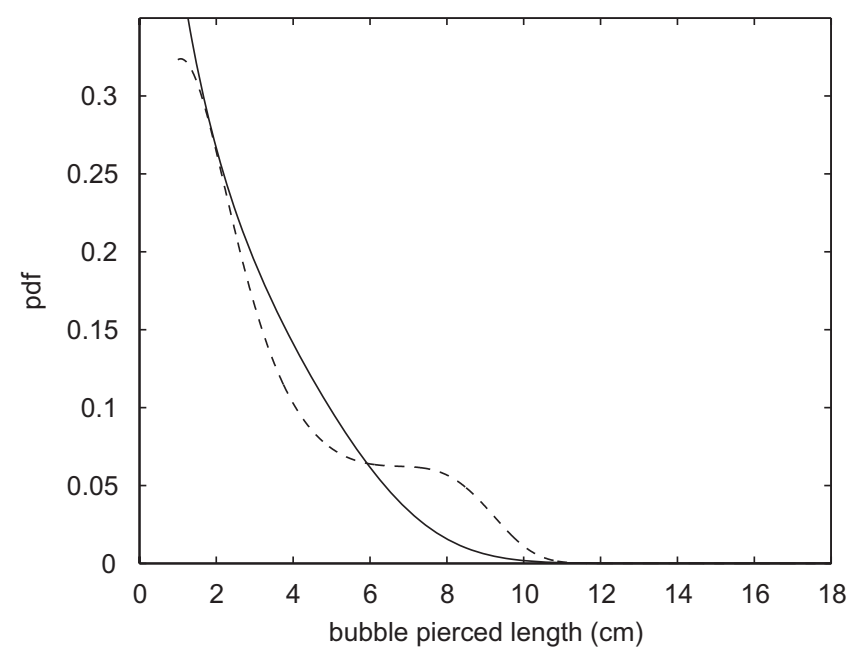

C



b

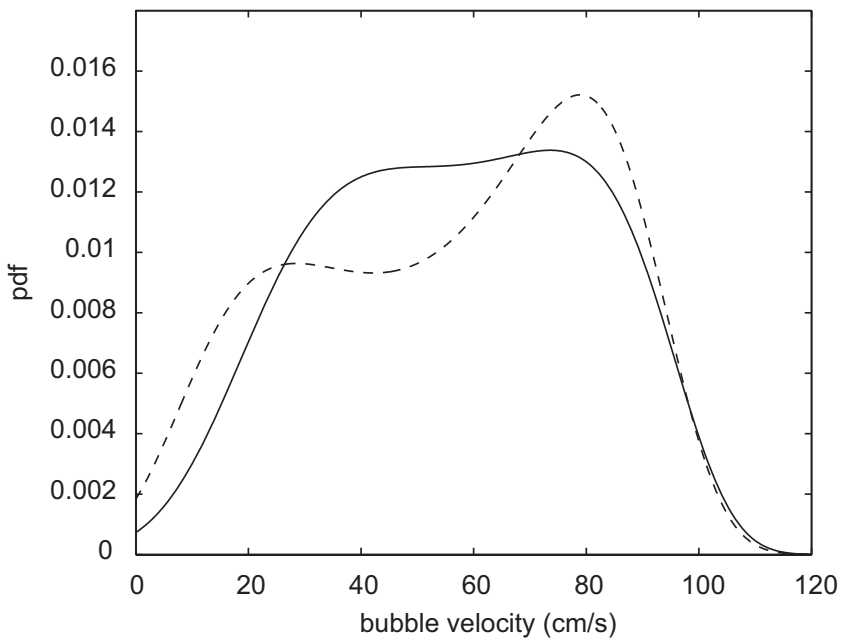

d



Fig. 10. PDF of (a) bubble pierced length, (b) bubble velocity, (c) bubble diameter $D$, and (d) volume equivalent diameter $D_{v}$, from optical (solid line) and pressure (dash line) measurements. $U=0.57 \mathrm{~m} / \mathrm{s}, U / U_{m f}=1.4 . z_{o p}=10.5 \mathrm{~cm}, z_{p t}=13 \mathrm{~cm} . r / R=0$.

Table 1

Mean and standard deviation of $y$ and $D_{v}$ of bubbles measured at $z=10.5 \mathrm{~cm}(r / R=0)$ and $z=5.5 \mathrm{~cm}(r / R=0.8)$

\begin{tabular}{lll}
\hline$z(\mathrm{~cm})$ & $y(\mathrm{~cm})$ & $D_{v}(\mathrm{~cm})$ \\
\hline $10.5(r / R=0)$ & Optical & Optical \\
& mean $=3.20$ & mean $=5.51$ \\
& std $=1.78$ & std $=2.38$ \\
& Pressure & Pressure \\
& mean $=3.72$ & mean $=6.49$ \\
& std $=2.36$ & std $=3.41$ \\
$5.5(r / R=0.8)$ & Optical & Optical \\
& mean $=2.82$ & mean $=4.75$ \\
& std $=1.43$ & std $=1.68$ \\
\hline
\end{tabular}

these distributions in Figs. 10(d) and 11(d), the number of bubbles with a diameter smaller than the minimum measurable diameter is expected to be very small.

Finally a sensitivity analysis was performed in order to study the influence of the geometry factor $Q$ in the calculation of the bubble size distribution. The maximum entropy method was used to calculate the PDF of $D_{v}$ from pierced length measurements for the case plotted in Fig. 10. The calculation was repeated for different values of the bubble geometry factor $Q$. Fig. 12(a) and (b) show, respectively, the mean and the standard deviation of these distributions as a function of the geometry factor $Q$, for the pressure and optical probe measurements. It can be seen that for $Q$ values around 0.5 , variations in the estimated shape factor of a $20 \%$ lead to variations of the estimated bubble diameter of around $5 \%$. However, the sensitivity of the size estimation is higher for a higher $Q$ and the variance of the distribution also increases. Nevertheless for the experimental conditions presented here, it will be seen that the influence of the geometry parameter $Q$ in the determination of the bubble size distribution is limited. Fig. 13 shows the factor $Q$ as a function of the superficial gas velocity and the height above the distributor obtained using the correlation (25), proposed by Werther (1976). In this figure it can be seen that, according to the sensitivity analysis and the range of experimental conditions shown in this work, the estimated $Q$ is around 0.55 and this value varies less than a $15 \%$ for this column size and fluidization regime. 
a



C

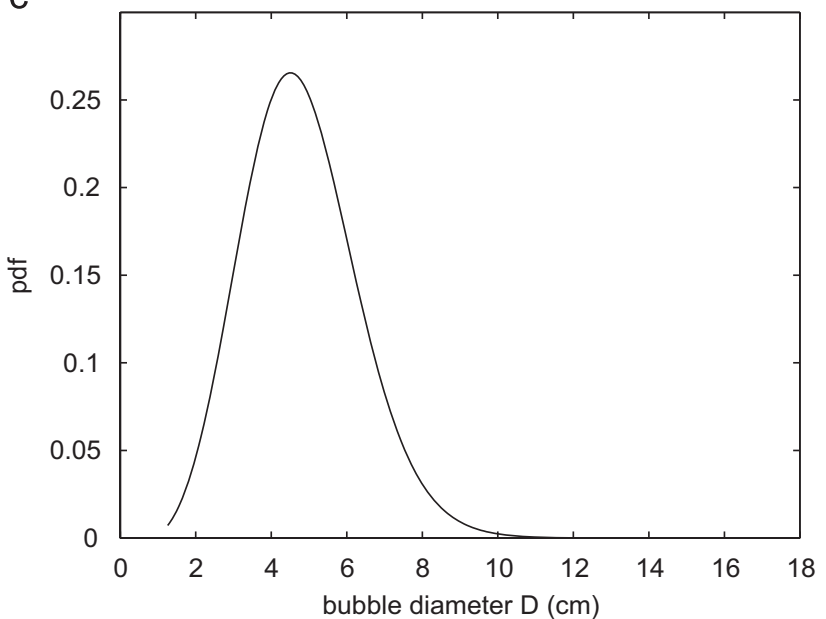

b

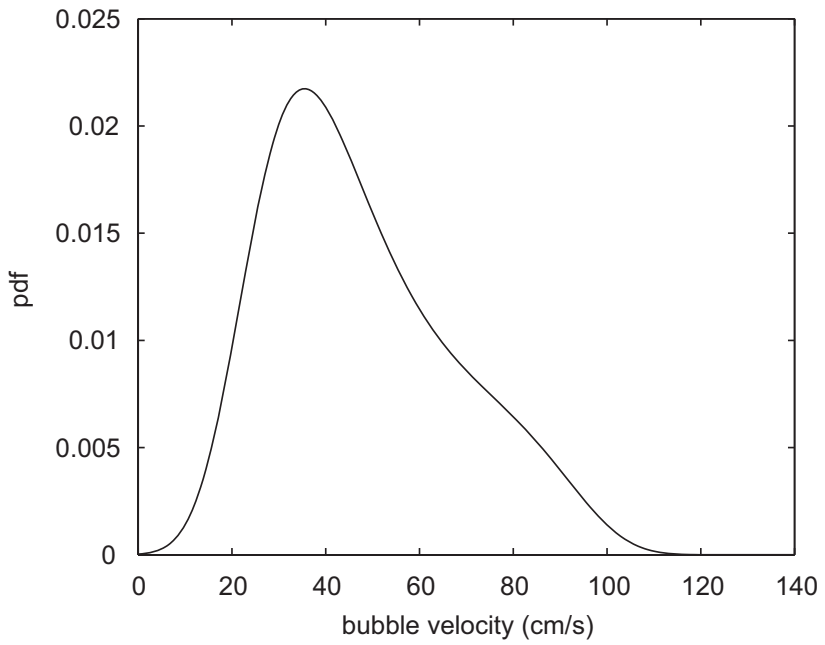

d



Fig. 11. PDF of (a) bubble pierced length, (b) bubble velocity, (c) bubble diameter $D$, and (d) volume equivalent diameter $D_{v}$ from optical measurements. $U=0.57 \mathrm{~m} / \mathrm{s}$, $U / U_{m f}=1.4 . z_{o p}=5.5 \mathrm{~cm} . r / R=0.8$.

a

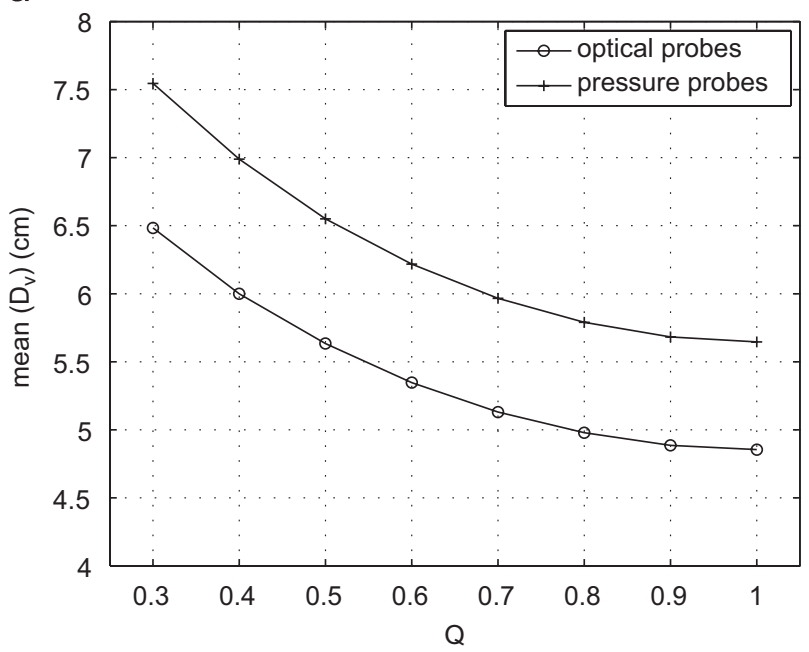

b

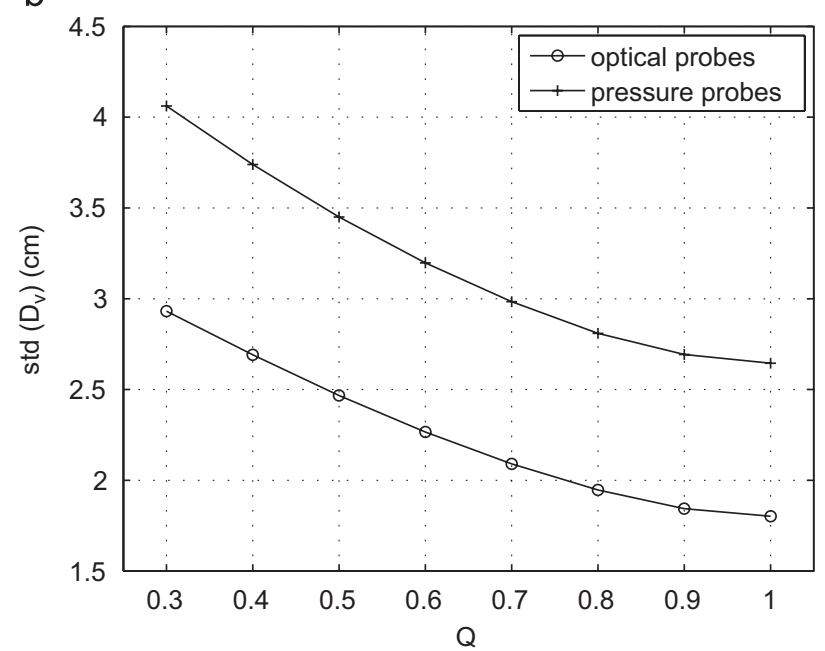

Fig. 12. (a) Mean and (b) standard deviation of the PDF of $D_{v}$ calculated with the maximum entropy method as, a function of the geometry factor $Q$ used to model the bubbles. $U=0.57 \mathrm{~m} / \mathrm{s}, U / U_{m f}=1.4 . z_{o p}=10.5 \mathrm{~cm}, z_{p t}=13 \mathrm{~cm} . r / R=0$. 


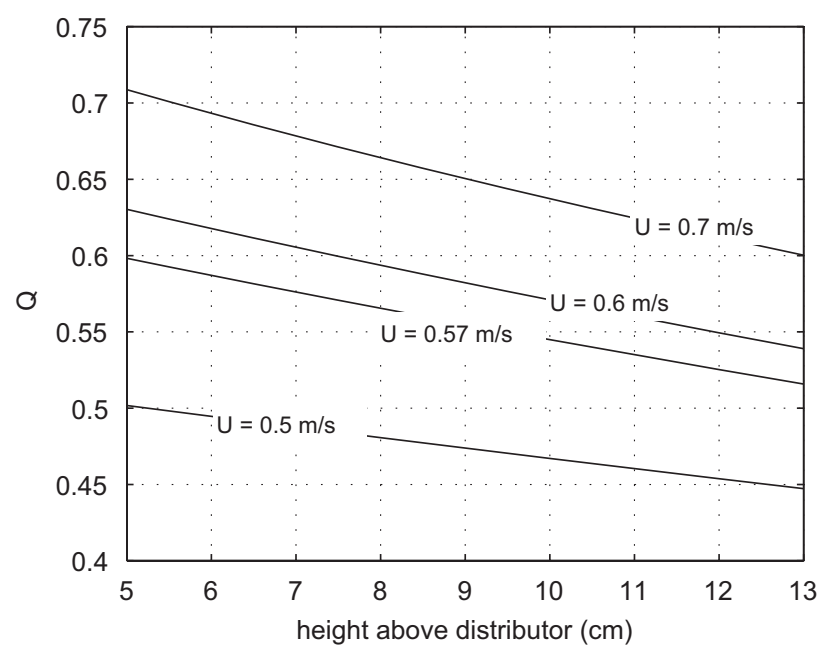

Fig. 13. Geometry factor $Q$ in Fig. 1 as a function of the gas superficial velocity and axial position calculated using correlation (25).

\section{Conclusions}

In this paper the maximum entropy principle has been applied to obtain the probability density distributions of bubble pierced length, bubble velocity and bubble diameter in fluidized beds. Bubble pierced length and velocity distributions were obtained directly from experimental measurements, while an estimation of the bubble diameter raw moments was inferred from pierced length measurements. The bubble geometry considered in this deduction was a truncated spheroid, which is the most general shape that can be considered for bubbles ascending in fluidized beds. Since the sampling probes do not allow to measure pierced lengths smaller than the distance between probes, $s$, this constraint was introduced in the size distribution estimation.

Experimental measurements of bubble pierced length and bubble velocity were carried out recording differential pressure and optical probe signals. The explained method was applied to these measurements in order to obtain the PDF of pierced length, velocity and diameter of the detected bubbles.

Optical probes have been found to be more flexible, being able to measure along the whole bed height. The optical signal is also less affected by the whole bed phenomena other than the bubble passage, and they discriminate between gas and particle phase more accurately. The pressure probe gave similar results to optical probes regarding mean values, but the variance of the distributions were higher due to the influence of other phenomena in the bed besides bubble passage. The analysis of differential pressure signal was not enough to distinguish two bubbles coalescing from a large bubble. It was not possible to measure the size and velocity of the bubbles close to the distributor from pressure probe measurements, most probably due to their small size. In any case, the height range where pressure signal can be used to obtain the bubble size is very dependent on the particular system that is being studied. However, pressure probes have been shown to provide reliable information on bubble characteristics in the middle of the bed, having the advantage of being very simple instrumentation easily implemented in hot devices and widely used in the industry.

\section{Notations}

D bubble diameter, $\mathrm{m}$

$D_{s} \quad$ diameter of the bubbles having $y>s, \mathrm{~m}$

$\Delta t$

$\Delta u_{b}$

$u_{b}$

$U$

$U_{m f}$

V

$V_{b}$

$x$

$y$

$\alpha_{1}$

$\alpha_{2}$

$\Delta$

$\varepsilon$

$\lambda$

$\rho$

$\rho_{\text {bulk }}$

$\rho_{f}$

$\rho_{p}$

$\phi$

\section{Subscripts}

1

2

\section{Greek letters}

bubble volume equivalent diameter, $\mathrm{m}$

lower limit of $D_{v}$ distribution, $\mathrm{m}$

relative error of the measured bubble velocity, $\mathrm{m} / \mathrm{s}$ relative error of the measured bubble pierced length, $\mathrm{m}$ function in Eq. (2)

order of the sample raw moments

optical probe signal, $\mathrm{V}$

gauge pressure, $\mathrm{Pa}$

differential pressure, $\mathrm{Pa}$

hydrostatic pressure, $\mathrm{Pa}$

probability density function, dimensionless

probability density function of the bubbles with $y>s$, dimensionless

bed pressure drop, $\mathrm{Pa}$

distributor pressure drop, Pa

bubble geometry factor, dimensionless

radial coordinate

distance between the probe and the bubble symmetry axis, $m$

maximum distance between the probe and the bubble symmetry axis for bubbles with $y>s, \mathrm{~m}$

bed radius, $m$

distance between the lower and the upper probes, $\mathrm{m}$

distance between probe ports, $\mathrm{m}$

parameter defined in Eq. (16)

time, $s$

time at which the bubble nose reaches the lower pressure probe, $s$

time at which the bubble nose reaches the upper pressure probe, $s$

time lag between the signals measured by the lower and the upper probes, $s$

time length corresponding to the bubble passage $\approx y / u_{b}$, $\mathrm{s}$

time between samples, $\mathrm{s}$

error of the measured bubble velocity, $\mathrm{m} / \mathrm{s}$

vertical velocity of bubbles, $\mathrm{m} / \mathrm{s}$

superficial gas velocity, $\mathrm{m} / \mathrm{s}$

minimum fluidization velocity, $\mathrm{m} / \mathrm{s}$

bubble volume, $\mathrm{m}^{3}$

volume of a sphere having the same diameter as the bubble, $\mathrm{m}^{3}$

generic variable in Eq. (2)

bubble pierced length, $\mathrm{m}$

error of the measured bubble pierced length, $m$

height above the distributor, $\mathrm{m}$

axial position of the optical probe, $m$

axial position of the pressure probe, $m$

bubble geometry factor, dimensionless

bubble geometry factor, dimensionless

integration domain, dimensionless

voidage, dimensionless

Lagrange multiplier

ratio of the volume of the bubble to the volume of a sphere with the same diameter $V / V_{b}$, dimensionless density of particle phase $=\left[\rho_{p}(1-\varepsilon)+\rho_{f} \varepsilon\right], \mathrm{kg} / \mathrm{m}^{3}$ density of fluidizing fluid, $\mathrm{kg} / \mathrm{m}^{3}$

particle density, $\mathrm{kg} / \mathrm{m}^{3}$

factor in Eq. (25), $\mathrm{m}^{-1}$

lower measuring probe

upper measuring probe 


\section{Acknowledgment}

This work has been supported by the national RDI plan of the Spanish Ministry of Science and Innovation under the project number ENE2006-01401.

\section{References}

Bai, B., Gheorghiu, S., van Ommen, J.R., Nijenhuis, J., Coppens, M.-O., 2005 Characterization of the void size distribution in fluidized beds using statistics of pressure fluctuations. Powder Technology 160, 81-92.

Chan, I.H., Sishtla, C., Knowlton, T.M., 1987. The effect of pressure on bubble parameters in gas-fluidized beds. Powder Technology 53, 217-235.

Cheremisinoff, N.P., 1986. Review of experimental methods for studying the hydrodynamics of gas-solid fluidized beds. Industrial \& Engineering Chemistry Process Design and Development 25, 329-351.

Clark, N.N., Atkinson, C.M., 1988. Amplitude reduction and phase lag in fluidized-bed pressure measurements. Chemical Engineering Science 43, 1547-1557.

Clark, N.N., Turton, R., 1988. Chord length distributions related to bubble size distributions in multiphase flows. International Journal of Multiphase Flow 14, 413-424.

Clark, N.N., Liu, W., Rurton, R., 1996. Data interpretation techniques for inferring bubble size distribution from probe signals in fluidized systems. Powder Technology 88, 179-188.

Davidson, J.F., Harrison, D., 1963. Fluidized Particles. Cambridge University Press, Cambridge.

Dent, D., LaNauze, R.D., Joyce, T., Fulford, V., Peeler, P., 1989. Differential pressure measurements-their application to measurements of fluidized bed combustion parameters. In: Proceedings of the 10th Conference on Fluidized Bed Combustion.pp. 451-456.

Ghadiri, M., Carter, B., Rathbone, R.R., Clift, R., Medhurst, S.J., Rogers, E.A. Summerfield, I., 1988. Diagnosis of gas flow patterns in fluidised beds. In: Proceedings of the 4th International Fluidized Bed Combustion Conference. Institute of Energy,

Hatano, H., Ishida, M., 1981. The entrainment of solid particles from a gas-solid fluidized bed. Journal of Chemical Engineering of Japan 14, 306-311.

Herringe, R.A., Davis, M.R., 1976. Gas-liquid mixture flows. Journal of Fluid Mechanics 73, 97-123.

Ishida, M., Shirai, T., 1980. Measurement of the velocity and direction of flow of solid particles in a fluidized bed. Powder Technology 27, 1-6.

Jaynes, E.T., 1957. Information theory and statistical mechanics. Physical Review $106,620-630$.

Kai, T., Misawa, M., Takahashi, T., Tiseanu, I., Ichikawa, N., Takada, N., 2000 Application of fast X-ray CT scanner to visualization of bubbles in fluidized bed. Journal of Chemical Engineering of Japan 33, 906-909.

Littman, H., Homolka, G.A.J., 1973. The pressure field around a two-dimensional gas bubble in a fluidized bed. Chemical Engineering Science 28, 2231-2243.

Liu, J., Grace, J.R., Bi, X.T., 2003. Novel multifunctional optical-fiber probe: I. Development and validation. A.I.Ch.E. Journal 49, 1405-1420.

Liu, W., Clark, N.N., 1995. Relationships between distributions of chord lengths and distributions of bubble size including their statistical parameters. International Journal of Multiphase Flow 21, 1073-1089.
Liu, W., Clark, N.N., Karamavruc, A.I., 1996. General method for the transformation of chord-length data to a local bubble size distribution. A.I.Ch.E. Journal 42, 2713-2720.

Liu, W., Clark, N.N., Karamavruc, A.I., 1998. Relationship between bubble size distribution and chord-length distribution in heterogeneously bubbling systems. Chemical Engineering Science 53, 1267-1276.

Luther, S., Rensen, J., Guet, S., 2004. Bubble aspect ratio and velocity measurements using a four-point fiber-optical probe. Experiments in Fluids 36, 326-333.

Mainland, M.E., Welty, J.R., 1995. Use of optical probes to characterize bubble behavior in gas-solid fluidized beds. A.I.Ch.E. Journal 41, 223-227.

Müller, C.R., Davidson, J.F., Dennis, J.S., Fennell, P.S., Gladden, L.F., Hayhurst, A.N., Mantle, M.D., Rees, A.C., Sederman, A.J., 2006. Real-time measurement of bubbling phenomena in a three-dimensional gas-fluidized bed using ultrafast magnetic resonance imaging. Physical Review Letters 96 154504-1-4.

Rockinger, M., Jondeau, E., 2002. Entropy densities with an application to autoregressive conditional skewness and kurtosis. Journal of Econometrics 106, 119-142.

Santana, D., Macías-Machín, A., 2000. Local bubble-size distribution in fluidized beds. A.I.Ch.E. Journal 46, 1340-1347.

Santana, D., Rodríguez-Rodríguez, J., Almedros-Ibáñez, J.A., Martínez-Bazán, C., 2006. Characteristics lengths and maximum entropy estimation from probe signals in the ellipsoidal bubble regime. International Journal of Multiphase Flow 32, 1123-1139.

Schweitzer, J.M., Bayle, J., Gauthier, T., 2001. Local gas hold-up measurements in fluidized bed and slurry bubble column. Chemical Engineering Science 56, 1103-1110.

Sellens, R.W., Brzustowski, T.A., 1985. A prediction of the drop size distribution in a spray from first principles. Atomization Spray and Technology 1, 89-102.

Sitnai, O., 1982. Utilization of the pressure differential records from gas-fluidized beds with internals for bubble parameters determination. Chemical Engineering Science 37, 1059-1066.

Turton, R., Clark, N.N., 1989. Interpreting probe signals from fluidized beds. Powder Technology 59, 117-123.

Vázquez, C., Nombela, J.L., Sobrino, C., de Vega, M., Zubía, J., Montero, D.S., 2007. Plastic fiber-optic probes for characterizing fluidized beds in bubbling regime. In: 16th International Conference on Plastic Optical Fiber (POF), pp. 202-205.

Venkata Ramayya, A., Venkateshan, S.P., Kolar, A.K., 1996. Estimation of bubble parameters from differential pressure measurements in gas-fluidized beds. Powder Technology 87, 113-126.

Werther, J., 1974a. Bubbles in gas fluidised beds-part I. Transactions of the Institution of Chemical Engineers 52, 160-169.

Werther, J., 1974b. Bubbles in gas fluidised beds-part II. Transactions of the Institution of Chemical Engineers 52, 149-159.

Werther, J., 1976. Convective solids transport in large diameter gas fluidized beds. Powder Technology 15, 155-167.

Werther, J., 1999. Measurements techniques in fluidized beds. Powder Technology 2, $15-36$.

Werther, J., Molerus, O., 1973. The local structure of gas fluidized beds-I. A statistically based measuring system. International Journal of Multiphase Flow $1,103-122$.

Yasui, G., Johanson, L.N., 1958. Characteristics of gas pockets in fluidized beds. A.I.Ch.E. Journal 4, 445-452.

Yates, J.G., Simons, S.J.R., 1994. Experimental methods in fluidization research. International Journal of Multiphase Flow 20(Suppl.), 297-330. 